\title{
Clinical and immunological assessment of HIV infection
}

A G Bird

\section{Introduction}

Human immunodeficiency virus (HIV) infection results in a progressive immunodeficiency primarily affecting $\mathrm{T}$ cell mediated immunity. It is also associated with direct damage to other organs including the brain and gastrointestinal tract which may be the direct result of either primary viral pathogenicity or a consequence of immunopathological effects. This review will cover only aspects of the clinical presentation of HIV infection resulting from the associated immunodeficiency and its laboratory assessment.

Accurate clinical staging is essential for epidemiological monitoring of the HIV epidemic, overall, in patient selection for clinical trials, and is of increasing importance in the assessment of individual patients for therapeutic intervention. Laboratory monitoring is gaining increasing importance in the initial staging of disease in individual patients, in the identification of immunological progression of those patients who remain asymptomatic, and in the recruitment to and assessment of clinical trials of new therapeutic agents against HIV infection.

\section{Staging of HIV disease}

In most individuals infected with HIV evidence of cellular immune deficiency appears after a latent period of good health that usually extends for a number of years after primary viral infection. Available evidence from several cohort studies suggests that 10 years after infection half of adults acquiring infection by any of the recognised routes will have developed AIDS and that most of the remainder will have either symptomatic disease which does not yet fulfill the case definition for AIDS or laboratory evidence of persistent virus infection and subclinical immune deficiency. ${ }^{1-4}$ However, in all studies of such duration there remains a residue of individuals with little clinical or laboratory evidence of cellular immunodeficiency. Whether this minority population identifies a group of individuals who will only show late evidence of progression or who genuinely represent individuals who are immune to the pathogenic potential of HIV will only become apparent with longer term follow up.

Clinical presentation and natural history of disease is less predictable and more diverse in children who have acquired the infection vertically during gestation or at childbirth, and is beyond the scope of this article. Readers are referred to review articles dealing specifically with paediatric aspects of HIV infection..$^{5-7}$

Because viral infection in adults, and the associated immunodeficiency, is progressive in most individuals, several clinical staging systems have been proposed which are essentially hierarchical and describe events which once occurred do not revert to earlier stages. However, with the increasing use of specific treatment in patients it is now possible in practice to return patients temporarily to earlier levels of such classifications. Such reversion is seen particularly in laboratory markers of immunological progression in patients receiving antiretroviral treatment.

The most widely adopted Centers for Disease Control (CDC) clinical staging system of $\mathrm{HIV}$ infection is reproduced in table 1 but may soon be replaced by a proposed CDC/WHO classification which will incorporate both laboratory and clinical markers (table 2). The proposed new classification recognises the importance of the long asymptomatic stage of disease in determining the eventual outcome of

Table 1 Centers for Disease Control (CDC) classification of HIV infection

\begin{tabular}{ll}
\hline Stage I & Acute HIV infection and seroconversion \\
Stage II & Asymptomatic HIV infection \\
Stage III & Persistent generalised lymphadenopathy \\
Stage IV & A Constitutional disease (old AIDS related \\
& B Neomplex (ARC)) \\
& C Sevelogical disease \\
C1 CDC AIDS immoleficiency & C2 severe infections outwith AIDS \\
definition & Decondary cancers within AIDS definition: \\
& Kaposi's sarcoma \\
& E Other conditions \\
\hline
\end{tabular}

Table 2 Proposed CDC/WHO classification system

\begin{tabular}{|c|c|c|}
\hline I & $\begin{array}{l}\text { Clinical: } \\
\text { Category A }\end{array}$ & $\begin{array}{l}\text { - Primary clinical HIV infection } \\
\text { (seroconversion illness) } \\
\text {-Asymptomatic HIV infection } \\
\text { - Persistent generalised lymphadenopathy } \\
\text { - Conditions indicative of HIV associated } \\
\text { cellular immune deficiency not listed in } \\
\text { Category C, such as, } \\
\text { - Bacterial pneumonia or meningitis } \\
\text { - Pharyngeal or vaginal candidiasis } \\
\text { - Cervical dysplasia or carcinoma } \\
\text { - Oral hairy leucoplakia } \\
\text { - Recurrent or multidermatomal herpes } \\
\text { zoster } \\
\text { - Pulmonary tuberculosis } \\
\text {-Idiopathic thrombocytopenic purpura } \\
\text { - Unexplained constitutional disturbance } \\
\text { - Old CDC stages IV B, C1, C2, D, E }\end{array}$ \\
\hline $\begin{array}{l}\text { (1) } \\
\text { (2) } \\
\text { (3) }\end{array}$ & $\begin{array}{l}\text { aboratory: } \\
\text { CD4 cells } \\
>0.5 \\
0.2-0.9 \\
<0.2\end{array}$ & $\begin{array}{l}\text { Total lymphocyte count } \times 10^{9} / l \\
>2 \\
<1-1 \cdot 9 \\
<1\end{array}$ \\
\hline
\end{tabular}

A G Bird

Correspondence to: Dr A G Bird

Accepted for publication 13 January 1992
HIV Immunology

Unit, Department of 
the disease process and the need to direct therapeutic trial intervention at this group of patients. It includes clinical and laboratory staging systems assessed in parallel to improve discrimination of disease stages and recognises the importance of laboratory markers of disease in the subclassification of asymptomatic patients. The addition of lymphocyte counts in this classification identifies the reality that in many countries reliable methodologies for accurate quantitation of CD4 counts, although desirable, are not routinely available.

\section{Acute (primary) HIV infection}

Acute HIV infection is identified as a clinical event in only about $15-20 \%$ of individuals. Defined as an acute viral illness resembling infections mononucleosis and less commonly associated with acute neurological manifestations, which include aseptic meningoencephalitis or neuropathy, these symptoms are temporally associated with the first appearance of HIV antibodies in infected individuals. ${ }^{8}$ This acute viral illness is self limiting in virtually all cases but if prolonged has been associated with more rapid subsequent progression to symptomatic immunodeficiency. ${ }^{9}$

\section{Asymptomatic HIV disease and persistent generalised lymphadenopathy} The proposed new category A of the CDC/ WHO staging system combines primary viral infection together with asymptomatic disease and persistent generalised lymphadenopathy (PGL) as a single staging entity. This summation is a recognition of the fact that natural history studies have indicated that asymptomatic HIV seropositive (PGL) subjects of known seroconversion date have no increased propensity to disease progression when compared with similar subjects without clinical lymphadenopathy. ${ }^{10}$ Most HIV seropositve individuals remain in category $\mathrm{A}$ for long periods of time and yet within this stage a wide range of immunological changes of different severity can be seen. It is in this category that the inclusion of immunological staging will add most additional information.

\section{Early manifestations of HIV associated immunodeficiency}

Symptomatic HIV immunodeficiency generally appears many years after initial viral infection. Most, but not all, patients pass through a sequence of mild and progressive constitutional disturbance frequently associated with minor opportunistic infections identified in category B. Few of these conditions are restricted to HIV infection and some can be frequently encountered in non-HIV infected individuals who are well or mildly immunocompromised. Oral hairy leucoplakia or recurrent or extensive herpes zoster are most typical of the earlier stage HIV immunodeficiency. Only unusually are they encountered in association with other diseases and only exceptionally in normal individuals. Pulmonary tuberculosis can obviously present in immunocompetent individuals but appears with increased frequency and severity at all immunological stages of HIV disease in areas of endemic infection.

Most of the conditions identified in category $B$ have been recognised for many years as being associated with cell mediated immune deficiency of any cause, and more than one of these conditions appearing together or sequentially should raise suspicion of underlying immune deficiency. Investigation must now also include assessment for and exclusion of HIV associated immunodeficiency. In contrast, bacterial infections with encapsulated species are more typically associated with antibody deficiency states but are also seen with increased frequency and severity in HIV seropositive subjects. They are particularly prevalent amongst populations of infected drug users where they can be the major cause of morbidity in early HIV infection. ${ }^{11}$

\section{Late HIV associated immunodeficiency}

Clinical presentations in this category are associated with profound immune deficiency and comprise a range of opportunistic organisms and tumours. Their character and severity of presentation are incompatible with general or immunological good health. Most presenting infections or tumours in this category were included in the original case definitions of AIDS. However, for several years this stage of disease has also included the severe constitutional symptoms of unexplained weight loss, diarrhoea, or fever previously known as AIDS related complex, an inevitable harbinger of symptomatic immune deficency. Infections in this category of disease are progressive without appropriate treatment. Some, notably Pneumocystis carinii, are now largely preventable with prophylactic treatment. This realisation is resulting in increasing enthusiasm for the earlier detection of HIV infection and the monitoring of individuals to determine those at greatest risk of short term progression, to whom new therapeutic approaches are being targeted. Together with the more effective therapeutic regimens for established opportunistic infections and the use of antiretroviral treatment, the length of survival of individuals in this category has been substantially lengthened from an initial mean of six months to two years or more. However, although longer survival is now generally achievable continued disease progression is inevitable in this category as a result of the severity and irreversibility of the underlying cellular immunodeficiency. With the increasing success in treatment of certain infections, there is a rising morbidity with opportunistic agents which are more difficult or impossible to treat (cytomegalovirus, atypical mycobacterial infections, or cyptosporidiosis) and a particular tendency for the development of invasive Kaposi's sarcoma or immunoblastic or Burkitt's type lymphomas as a terminal event. Although lymphomas are reported often $(30 \%)$ in patients who have been receiving long term antiretroviral treatment for periods in excess of two years, ${ }^{12}$ this 
probably represents a feature of severe long term immune deficiency rather than a primary consequence of treatment with the nucleoside analogue class of drugs.

\section{Immunological assessment of HIV infection}

A more complete description of the clinical natural history of HIV infection has emerged from the long term follow up of infected subjects, from documented episodes of seroconversion. Such studies have indicated that HIV infection is a predictably progressive disease with a mean incubation time to severe symptomatic disease of about 10 years in young adults, irrespective of mode of acquisition of the infection. ${ }^{34}$ This finding, combined with the results of studies and trials suggesting that prophylactic treatment can delay some of the later opportunistic infections, and that specific antiretroviral treatment can slow the rate of disease progression over intermediate time periods, have forced a re-evaluation of the need for earlier identification of HIV infection and the clinical and immunological assessment and follow up of the asymptomatic subject.

Following the first detection of HIV infection, the immune status of every individual should be assessed to guide clinical and therapeutic decision making. This is forcing reappraisal of the long asymptomatic phase of the disease and is the reason for the introduction of immunological subdivisions of this stage of disease.

Increasing experimental evidence suggests that the early stages of HIV infection are generally associated with a strong and partially effective anti-HIV immune response which is associated with the reduction of initially high levels of virus replication. ${ }^{13}$ This state of relative immunity can persist for many years but is apparently eroded by the ease with which HIV mutates away from effective specific antibody and cellular responses. ${ }^{14}$ However, the observations from natural history studies which suggest that immune responses can effectively suppress viral replication in the early stages of infection for many years gives hope that earlier therapeutic intervention may be associated with considerably enhanced survival.

Many immunological parameters are influ-

Table 3 Characteristics of markers of potential value in assessment of asymptomatic HIV disease

\begin{tabular}{lllll}
\hline & $B_{z} M$ & CD4 count & p24 antigen & Viral isolation \\
\hline $\begin{array}{c}\text { Markers present in most } \\
\text { infected patients } \\
\text { (sensitivity) }\end{array}$ & No & Yes & No & Yes \\
$\begin{array}{c}\text { Marker absent from } \\
\text { non-progressor patients } \\
\text { (specificity) }\end{array}$ & Yes & Yes & Yes & No \\
$\begin{array}{c}\text { Change in marker associated } \\
\text { with disease progression }\end{array}$ & In some & Yes & In some & If quantitated \\
$\begin{array}{c}\text { Marker influenced by } \\
\text { treatment }\end{array}$ & Yes & Yes & If present & $?$ \\
$\begin{array}{c}\text { Marker directly related to } \\
\text { disease pathogenesis }\end{array}$ & No & Yes & If present & Yes \\
$\begin{array}{c}\text { Assay standardisable with } \\
\text { quality control }\end{array}$ & Yes & Yes & Yes & $?$ \\
$\begin{array}{c}\text { Widely available in clinical } \\
\text { centres }\end{array}$ & Yes & Yes & Yes & No \\
\hline
\end{tabular}

enced by HIV infection. In the late stages of disease virtually every available investigation is abnormal. As the priority for clinical assessment is to identify who is most likely to progress among a pool of asymptomatic individuals and how often follow up assessments are required, markers must be chosen which have the characteristics given in table 3 .

Experience from the classification and assessment of primary immune deficiency states has indicated that absence of cell populations responsible for immune recognition or effector responses is usually associated with clinically overt immunodeficiency. However, severe infections can also be seen in association with conditions in which loss of specific immune responses is not necessarily associated with deletion of a total cell population. Applying these observations to HIV infection means that the deletion of the CD4 cell population, which characterises the late stages of HIV infection, is associated with severe clinical immunodeficiency and complete loss of in vitro $\mathrm{T}$ cell immune responses. However, in early HIV infection loss of specific functional $\mathrm{T}$ cell responses generally precede overt evidence of total CD4 population depletion. There is, therefore, increasing interest in examining specific immune responses to characterise early evidence of immune compromise in asymptomatic patients to identify those individuals at increasing risk of short term progression. $^{15}$

However, such approaches have three major difficulties. Assays require tissue culture of fresh blood lymphocytes, techniques which are difficult to apply on a large scale; particularly as containment facilities are required for such work. Secondly, such assays are difficult to standardise and quality control. Finally, the choice of antigen or polyclonal activator is difficult. If opportunistic organism antigens are used (the most physiologically relevant approach) then comparison of patients is a problem because it is likely that environmental exposure varies from person to person. For these reasons functional assays have not been widely evaluated in the longitudinal follow up of individual patients.

\section{Lymphocyte phenotypic markers}

Laboratory assesment of the degree of immunodeficiency generally includes quantitation of T cell subpopulations. Serum or cellular markers of immune activation give additional information. Because the destruction of the CD4 population is the central lesion of HIV associated immunodeficiency, severe depletion of this $T$ cell subpopulation is an invariable counterpart of the late stage disease characterised by major opportunistic infections. Moreover, several cohort studies have identified that a decline in the CD4 population in HIV seropositive subjects usually identifies a subgroup individual at high risk of short term progression (table 4). In contrast, individuals with persistently normal or stable CD4 populations have low rates of short term disease progression. 
Table 4 Incidence of AIDS in follow-up cohort studies from presentation CD4 count

\begin{tabular}{llll}
\hline Group & $\begin{array}{l}\text { Presentation } C D 4 \\
\text { count }\end{array}$ & $\begin{array}{l}\text { \% AIDS incidence at } \\
3 \text { years }\end{array}$ & Reference \\
\hline Homosexual & $<200$ & 87 & 3 \\
& $201-400$ & 46 & \\
Homosexual & $>400$ & 16 & 18 \\
& 242 & 68 & \\
Injecting drug user & $243-345$ & 23 & 19 \\
& $>496-490$ & 11 & \\
& $<200$ & 100 & \\
& $201-500$ & 51 & \\
\hline
\end{tabular}

These cohort observations have been largely responsible for recommendations that patients with low presentation CD4 counts or whose counts fall to low levels on sequential follow up should receive primary prophylaxis against $P$ carinii infection, the most common serious opportunistic pathogen in late stage HIV infection. Current advice is that all adults with CD4 counts consistently below $0.2 \times 10^{9} / 1$ should receive such prophylaxis. ${ }^{16}$

Sequential follow up of asymptomatic patients has indicated that in most HIV seropositive subjects the CD4 count falls progressively with time at an overall rate of about $0.8-1.0 \times 10^{9} / 1 /$ year in cohorts. ${ }^{4}$ However, the rate of fall varies considerably from one individual to another. Experience suggests that individuals with a rapid rate of CD4 cell loss are more at risk of early clinical progression. The relative predictability of clinical progression in individuals showing sequential CD4 cell loss has led to increasing interest in directing specific antiviral treatment at this subgroup of individuals, although formal trial evidence that early treatment of such asymptomatic subjects results in prolonged overall survival is currently lacking.

Lymphocyte subpopulation numbers in blood are notoriously labile, being profoundly influenced by factors which include diurnal rhythm, stress, and intercurrent infection. ${ }^{17}$ Such considerations determine that trends of CD4 count stability or progression can only be assessed after a number of estimations have been obtained from asymptomatic individuals over a number of follow up attendances. Until a number of data points have been obtained, any assessment based on laboratory investigations alone is unreliable. This consideration provides a strong argument for the early identification and long term regular followup of the HIV infected subject. During this stage of the disease assessment of $\mathrm{T}$ cell markers and CD4 counts, in particular, should only be performed when patients do not have intercurrent infections when spuriously low and misleading values can be obtained.

CD8 counts are also abnormal in most patients with HIV infection. However, changes in CD8 counts are biphasic. Initial very high absolute numbers, which are associated with the immune response to primary HIV infection, gradually fall towards the normal range many years later, continuing to fall to subnormal concentrations in late stage disease. The dynamics of change in CD8 count make it unreliable as a marker in individual patients.

The divergent trends of CD4 and CD8 populations in early HIV disease has resulted in enthusiasm for use of the CD4:8 ratio in some centres. However, as the low ratio characteristic of most stages of HIV disease is also seen in several other viral infections and chronic immunological diseases, overreliance on this marker should not be encouraged. Absolute $\mathrm{T}$ cell subpopulation numbers provide more reliable staging indices.

\section{Immunological activation markers}

Activation of lymphocytes, especially CD8 cells, is a consistent feature of many primary or persistent viral infections. In HIV infection such activation persists through all stages of the disease and affects other lymphoid populations, including B cells. Several markers of activation on CD8 cells have been examined, including expression of class II histocompatibility antigens (DR), interleukin 2 receptors (CD25), and a number of cell adhesion molecules. Several of these show promise, although they have not been fully evaluated in the staging of individual patients or in the assessment of response to treatment.

Immunological activation results in the appearance of several lymphocyte and monocyte/macrophage products in serum or other body fluids. They include cytokines, immunoglobulins, $\beta_{2}$ microglobulin $\left(B_{2} M\right)$ and neopterin. Their relative ease of measurement combined with the potential for retrospective analysis on stored serum samples has resulted in considerable interest. Used alone, such markers perform only moderately well partly because of low sensitivity and because levels can also be strongly influenced by other causes of immune stimulation, of which the infections complicating the later stages of HIV infection are obvious examples. However, a number of studies have shown that when used together with CD4 counts $\beta_{2}$ microglobulin or neopterin provide additional staging information in individual patients. ${ }^{18-20}$ Raised concentrations provide additional power to identify the subgroup of asymptomatic patients at high risk of clinical progression. Equally significantly, low concentrations of $\beta_{2}$ microglobulin, when associated with relatively normal CD4 counts, identify a subgroup of patients at very low risk of short term clinical progression. Preliminary evidence suggests that $B_{2} M$ also has potential as a surrogate marker to assess early benefit from HIV specific treatment. ${ }^{21}$

Titres of total immunoglobulin classes rise differentially with different disease stages. Titres of IgA rise with progression to late stage disease but too late in most individuals to be of value in asymptomatic staging.

\section{Virological markers}

Methodology which permits quantitation of cell or plasma HIV viral load will be valuable in the assessment of disease and treatment. ${ }^{22} 23$ However, such techniques are expensive, poorly standardised, and not yet widely available. 
P24 antigen, the only widely applied viral marker, performs poorly in the asymptomatic patient where it would be of greatest value as most such individuals have no detectable antigen. Even in advanced disease, the determination of p24 has poor sensitivity for disease progression. Longer experience suggests that this marker does justify the great enthusiasm with which it was initially associated.

\section{Recommendations for frequency of clinical and laboratory monitoring} All identified HIV infected subjects should be offered regular clinical and laboratory follow up, irrespective of stage of disease presentation. Progress in understanding of disease natural history and evaluation of new treatments will require close study of the early stages of HIV infection.

Initial clinical assessment of asymptomatic subjects should be accompanied by baseline immunological investigation. In view of the inherent biological fluctuations associated with the CD4 count, a further count, irrespective of the initial one, is advisable within three months to establish baseline values against which subsequent trends can be assessed. Such trends cannot be extrapolated on the basis of single values for similar reasons and thus all apparent changes in $\mathrm{CD} 4$ values should be confirmed before clinical decisions are taken. For this reason it is advised that the asymptomatic individual is assessed at a minimum of six monthly intervals. Evidence of change will require more frequent evaluation. $\beta_{2}$ microglobulin and neopterin concentrations should be measured less frequently. As such markers are less labile (but also less sensitive to change) they should be assessed about once a year.

In later stage disease immunological markers become progressively less valuable as end-stage progression approaches. In such patients clinical assessment of symptomatology and laboratory diagnosis of infectious complications are the major priorities. Once the $\mathrm{CD} 4$ count has fallen consistently below $0.5 \times 10^{9} / 1$ in any individual then further assessment is probably only justifiable to assess therapeutic response to new antiviral agents.

\section{Future prospects}

The new CDC/WHO classification of HIV disease will probably change perceptions as attention is shifted from late to early stage disease. Moreover, the pace of assessment of new therapeutic approaches is already slowed by the widespread introduction of partially effective treatments in later stage disease, delaying the appearance of clinical endpoints in clinical trends. Future clinical trials are likely to become increasingly reliant on the enrolment of asymptomatic patients with assessment of efficacy based on the use of surrogate immunological or virological markers. Following initial evaluation of such trials it is likely that such laboratory monitoring will become increasingly valuable in the clinical decisions about when such agents should be used. For any such laboratory investigations to be widely acceptable internal and external quality control and standardised methodologies will be essential prerequisites.

1 Goedert JJ, Biggar RJ, Melbye M, et al. Effect of T 4 count and cofactors on the incidence of AIDS in homosexual men infected with human immunodeficiency virus. fAMA 1987;257:331-4

2 Eyster ME, Ballard JD, Gail MH, et al. Natural history of immunodeficiency virus infection in haemophiliacs: effects of T cell subsets, platelet counts and age. Ann Intern Med 1987;107:1-6.

3 Moss AR, Bacchetti P, Osmond D, et al. Seropositivity from HIV and the development of AIDS or AIDS related condition: three year follow up of the San Francisco condition: three year follow up of the San Francisc

4 Philips A, Lee CA, Elford J, et al. Prediction of progression to AIDS by analysis of CD4 lymphocyte counts in a to AIDS by analysis of CD4 lymphocyte

5 Bernstein LJ, Bye MR, Rubinstein A. Prognostic factors and life expectancy in children with acquired immunodeficiency syndrome and Pneumocystis carinii pneumonia. Am $\mathcal{F}$ Dis Child 1989;143:775-8.

6 Leibovitz E, Rigaud M, Pollack $\mathrm{H}$, et al. Pneumocystis carinii pneumonia in infants infected with the human immunodeficiency virus with more than $450 \mathrm{CD} 4 \mathrm{~T}$ lymphocytes per cubic millimeter. $N$ Engl f Med 1990; 323;531-3.

7 CDC Guidelines for prophylaxis against pneumocystis carinii pneumonia for children infected with human carinii pneumonia for children infected with hu

8 Cooper DA, Tindall B, Wilson EJ, Imrie AA, Penny $\mathrm{R}$ Characterization of $T$ lymphocyte responses during primary infection with human immunodeficiency virus. $\mathscr{f}$ Infect Dis 1988;157:889-96.

9 Pedersen C, Dickmeiss E, Gaub J, et al. T cell subse alterations and lymphocyte responsiveness to mitogen and antigen during severe primary infection with HIV: case series of seven consecutive HIV seroconverters. AIDS 1990;4:523-6.

10 Kaplan JE, Spira TJ, Fishbein DB, et al. A six-year follow-up of HIV infected homosexual men with lymphadenopathy. ҰAMA 1988;260:2694-7.

11 Brettle RP, Leen CLS. Natural history of HIV infection. In Bird AG, ed. Immunology of HIV infection. Amsterdam: Kluwer, 1991:1-30.

12 Pluda JM, Yarchoan R, Jaffe ES, et al. Development of nonHodgkins lymphoma in a cohort of patients with severe human immunodeficiency virus (HIV) infection on longterm antiretroviral therapy. Ann Intern Med 1990;113 276-82.

13 Daar ES, Moudgil T, Meyer RD, Ho DD. Transient high levels of viraemia in patients with primary human immunodeficiency virus type I infection. $N$ Engl $\mathcal{F} \mathrm{Med}$ 1991;324:961-4.

14 Philips RE, Rowland-Jones S, Nixon DF, et al. Human immunodeficiency virus genetic variation that can escape cytotoxic T cell recognition. Nature 1991;354:453-9.

15 Gruters RA, Miedema F. Lymphocyte functional analysis in HIV infection: mechanisms and clinical relevance. In Hird AG, ed. Immunology of HIV infection. Amsterdam: Bird AG, ed. Immuno

16 Masur H, Ognibene FP, Yarchoan R, et al. CD4 counts as predictors of opportunistic pneumonias in human immunodeficiency virus (HIV) infection. Ann Intern Med 1989;111:223-31.

17 Bird AG. Monitoring of disease progression of HIV infection. In: Bird AG, ed. Immunology of HIV infection. London: Kluwer, 1991:91-112.

18 Fahey JL, Taylor JMG, Detels $R$, et al. The prognostic value of cellular and serologic markers in infection with human immunodeficiency virus type I. N Engl F Med 1990;322 166-72.

19 Fernandez-Cruz E, Desco M, Montes MG, et al. Immunological and serological markers predictive of progression to AIDS in a cohort of HIV infected drug users. AIDS 1990;4:987-94.

20 Anderson RE, Lang W, Shiboski S, et al. Use of $B_{2}$ microglobulin level and CD4 count to predict development of acquired immunodeficiency syndrome in patients with human immunodeficiency virus infection. Arch Intern Med 1990;15:73-7.

21 Jacobsen MA, Bacchetti P, Kolokathis A, et al. Surrogate markers for survival in patients with AIDS and AIDS related complex treated with Zidovudine. $B M^{f}$ 1991;302:73-8.

22 Ho DD, Moudgil T, Alam M. Quantitation of the human immunodeficiency virus type 1 in the blood of infected persons. N Engl f Med 1989;321:1621-5.

23 Holodniy M, Katzenstein DA, Israelski DM, Merigan TC. Reduction in plasma HIV-RNA after dideoxynucleoside therapy as determined by the polymerase chain reaction. f Clin Invest 1991;88:1755-9. 